Document downloaded from:

http://hdl.handle.net/10251/88386

This paper must be cited as:

Esteve Bosch, R.; Toledo Alarcón, JF.; Rodriguez, J.; Querol, M.; Alvarez, V. (2016).

Readout and Data Acquisition in the NEXT-NEW Detector based on SRS-ATCA. Journal of Instrumentation. 11(1):1-8. doi:10.1088/1748-0221/11/01/C01008.

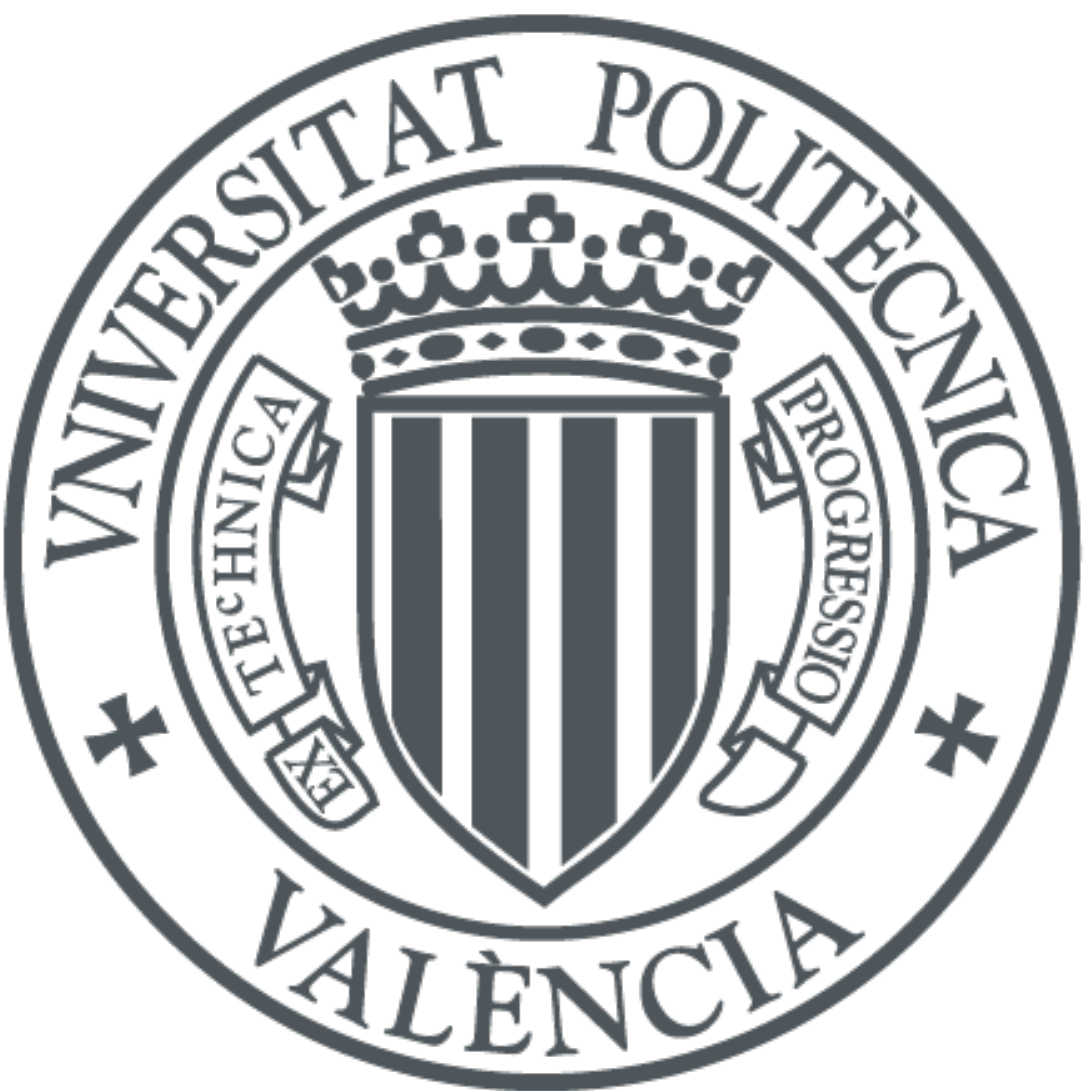

The final publication is available at

http://doi.org/10.1088/1748-0221/11/01/C01008

Copyright IOP Publishing: Hybrid Open Access

Additional Information 


\title{
1 Readout and Data Acquisition in the NEXT-NEW 2 Detector based on SRS-ATCA
}

\author{
R. Esteve ${ }^{\mathrm{a}, *}$, J. Toledo $^{\mathrm{a}}$, J. Rodríguez $^{\mathrm{b}}$, M. Querol $^{\mathrm{b}}$ and V. Álvarez ${ }^{\mathrm{b}}$ \\ ${ }^{a}$ Instituto I3M, Universitat Politècnica de València, Valencia, Spain \\ ${ }^{b}$ Instituto de Física Corpuscular, CSIC-UV, Valencia, Spain \\ E-mail: rauesbos@eln.upv.es
}

\section{ABSTRACT:}

The Scalable Readout System (SRS) was defined by the CERN RD51 Collaboration as a multichannel, scalable readout platform for a wide range of front ends. In 2014, SRS was ported to the ATCA (Advanced Telecommunications Computing Architecture) standard.

NEXT is an underground experiment aimed at searching for neutrinoless double-beta decay. NEXT-DEMO, a small-scale demonstrator, was read-out using SRS. NEXT has adopted SRSATCA for its first stage, called NEXT-NEW. Our presentation will describe the readout, DAQ and trigger for NEXT-NEW based on SRS-ATCA. This is, to our knowledge, the first experiment operating entirely on SRS-ATCA.

KEYWORDS: Data acquisition circuits, FPGA, ATCA.

\footnotetext{
* Corresponding author.
} 
1.1 ATCA in high-energy and nuclear physics

1.2 The Scalable Readout System (SRS) 2

2. SRS-ATCA implementation $\quad 2$

\section{Introduction}

\subsection{ATCA in high-energy and nuclear physics}

ATCA (Advanced Telecom Computing Architecture) is a PICMG 3.x standard that provides a reliable and scalable switched-based architecture to build data acquisition (DAQ) and trigger systems. It allows large-enough modules $(7.25 \mathrm{U}$ x $230 \mathrm{~mm})$, adequate power consumption (cooling is designed for at least $200 \mathrm{~W}$ per module) and large interconnection bandwidth (the majority of recent implementations use $10 \mathrm{~Gb} / \mathrm{s}$ channels, each consisting of four differential pairs) for today's high-energy and nuclear physics experiments. A brief discussion of the ATCA standard and its potential application to real-time systems is presented in [1]. More specifically, its suitability for the LHC DAQ upgrade was pointed out in [2].

Most ATCA implementations define FPGA-based processing carrier boards that accept I/O from several mezzanine boards in the front panel. These contain transceivers, frequently FPGAs and fast memories in some cases. Additionally, each carrier module can have an optional rear transition module (RTM) to provide additional I/O from the rear of the chassis. Boards are interconnected via high-speed serial backplanes implementing either a star topology (one slot in the chassis acts as a switch and each of the other modules provides a 4-pair channel to it), or more frequently, a full mesh (all slots provide one channel to every other slot). Neighbouring modules have dedicated interconnection channels in the backplane.

Among other features, ATCA chassis provide clocking, shelf management, hot swap capabilities and optional redundant cooling and power supplies. ATCA modules communicate with the shelf manager using the Intelligent Platform Management Interface (IPMI). This is known to be complex to implement, posing a non-negligible overhead and usually requiring a dedicated microcontroller.

Two representative examples of the current trend are described below. The first example is the Computing Node for the Belle II pixel detector DAQ and data reduction system [3]. In one of the two board versions, an ATCA carrier module accommodates 4 AMC mezzanines, each housing an FPGA, DDR2 memory, two SFP optical links (input data flow) and a Gbit Ethernet connector (output data flow). The carrier board provides a full-mesh interconnection (using $\leq$ 
6.5 Gb/s transceivers) between the four FPGAs in the mezzanines and a fifth FPGA in the carrier board, the latter being responsible to communicate with a full-mesh backplane in the ATCA chassis.

The second example is the Pulsar II ATCA carrier board [4] developed for the ATLAS Fast Tracker Data Formatter system, a trigger application. The full-mesh connectivity in the ATCA backplane is used to transfer time multiplexed event data from input boards to multiple track processing engines. The carrier board accommodates four mezzanine cards and two processing FPGAs (only one in the later Pulsar IIb revision), each with a dedicated DDR3 memory. The FPGAs directly interface the backplane using its embedded $10 \mathrm{~Gb} / \mathrm{s}$ transceivers instead of a dedicated switch IC, thus allowing custom efficient protocols (this solution is also found in [3]). A RTM provides expanded I/O via 14 pluggable QSFP+ and SFP+ transceivers for an aggregate bandwidth of $380 \mathrm{Gbps}$.

In both cases, as in many others, multi-gigabit full-mesh connectivity was key to the choice of ATCA as DAQ/trigger architecture.

\subsection{The Scalable Readout System (SRS)}

The Scalable Readout System (SRS) is an IP co-owned by CERN, Universitat Politècnica de València and IFIN-HH Bucharest. It was defined by the CERN RD51 Collaboration [5-6] as a scalable, multi-channel readout platform intended for small set-ups and medium-scale experiments. It was first conceived in 2009 with micro-pattern gaseous detectors in mind, though its flexibility allows targeting any kind of front-end. SRS has two main building blocks:

1. The Front-End Concentrator card (FEC) [7] is a flexible front-end interface and data concentrator. This 6U-height FPGA-based card processes data coming from edgemounted add-in cards. A variety of add-in cards exist with ADC channels, LVDS interfaces, or more specific front-ends like APV25, Beetle, VFAT or VMM2 ASICs. In the front panel, a number of connectors provide power, two SFP+ transceivers (usually Gbit Ethernet), NIM I/O and auxiliary RJ-45 sockets. The later allow implementing custom links [8] named DTCC carrying clock, slow controls, trigger and data over copper.

2. The Scalable Readout Unit (SRU) is a second data concentrator stage intended for larger scale applications.

Both modules are based on FPGA and can interface a DAQ PC farm via GbE links, thus reducing the DAQ and trigger systems to a network-based architecture. Many research institutions worldwide have successfully used SRS, including our first detector prototype (NEXT-DEMO) [9].

\section{SRS-ATCA implementation}

In 2014, SRS was ported to the ATCA (Advanced Telecommunications Computing Architecture) standard upon agreement with the German company EicSys. The use of certified crates with built-in and redundant cooling, power and shelf management makes it a more robust mechanical and electrical solution for prolonged operation in experiments than the original SRS flavour based on light Eurocard crates. Higher data bandwidth is achieved by replacing the DTCC link cables with multi-gigabit channels across a full-mesh backplane, enabling $10 \mathrm{~Gb} / \mathrm{s}$ I/O through the RTM and using faster FPGA and memory.

Figure 1 shows a picture of the 2-slot ACTA chassis provided by EicSys that has been used in the first tests of the NEXT-NEW experiment. 


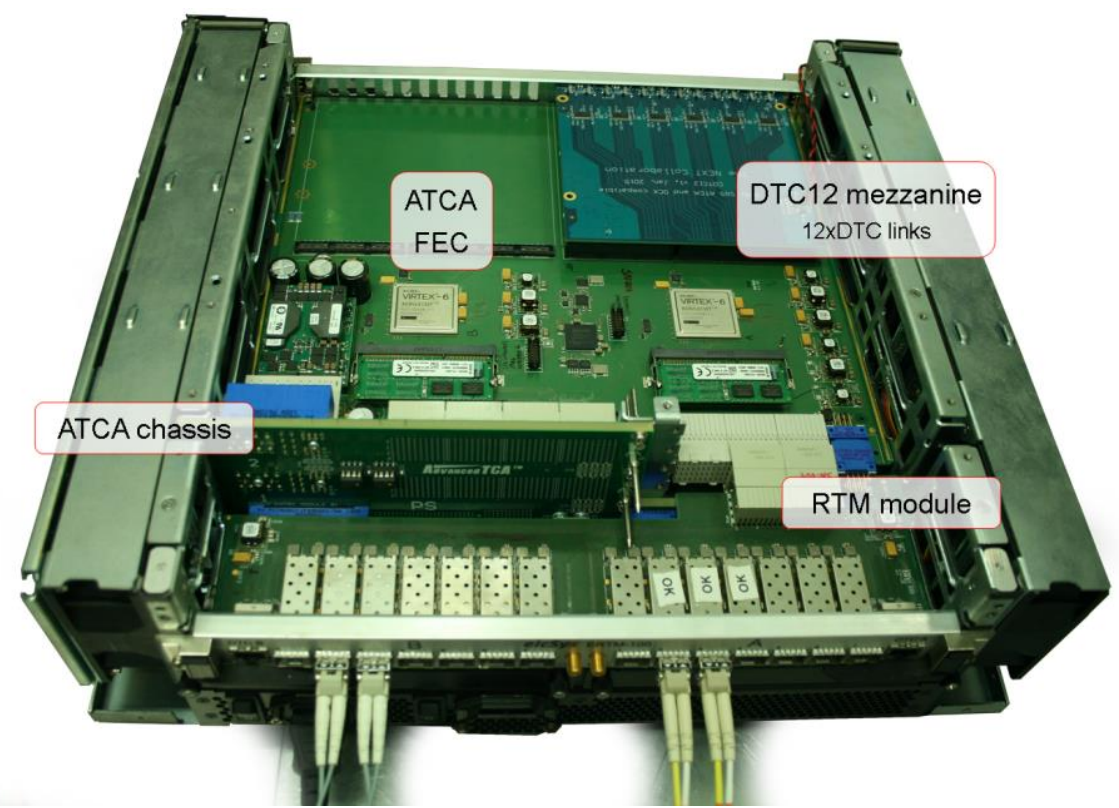

101 Figure 1. A two-slot ATCA chassis used in initial tests.

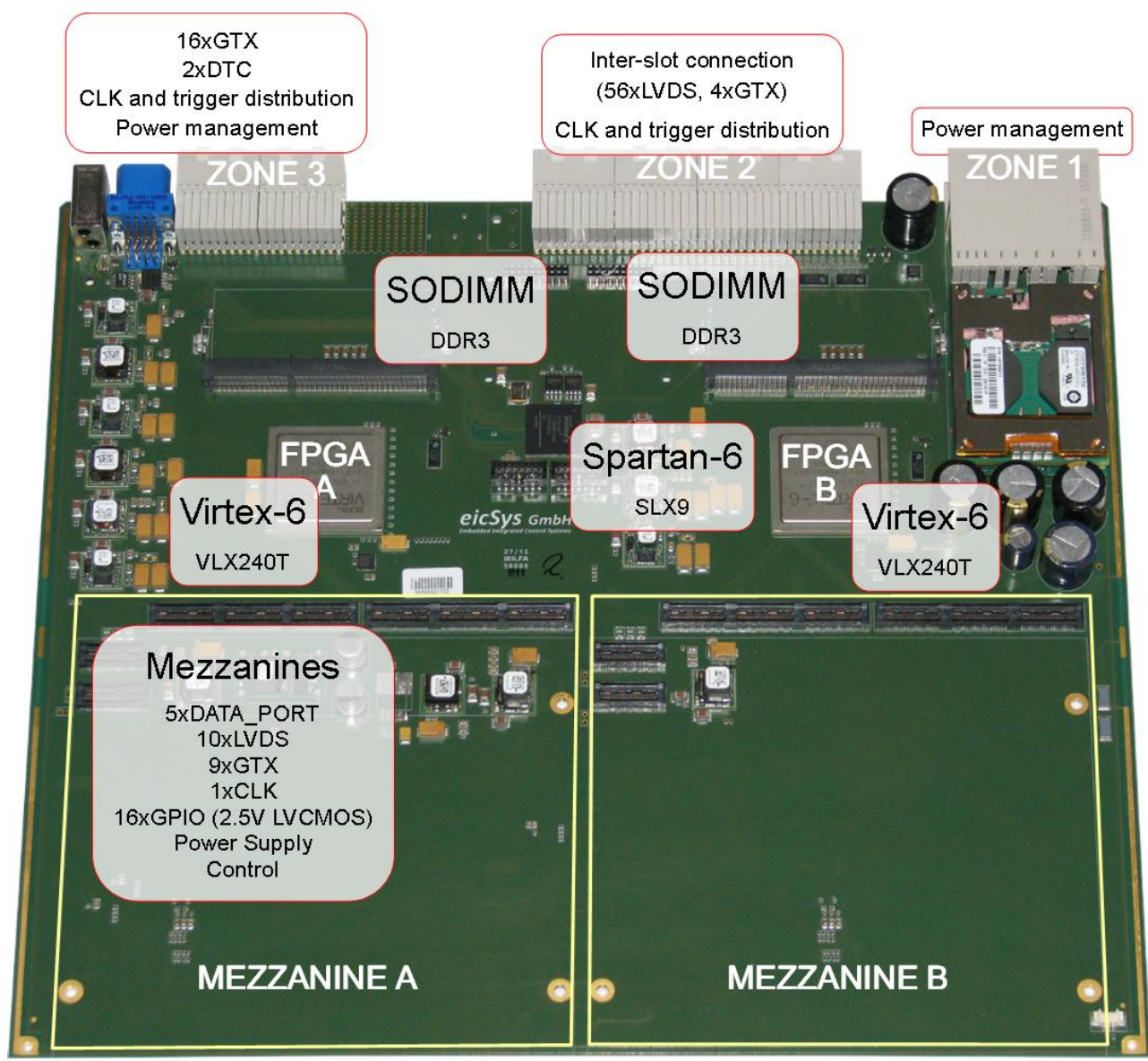

Figure 2. ATCA FEC module (2015 version). 
The ATCA-FEC carrier (figure 2) has two Xilinx Virtex-6 FPGAs (XC6VLX240T1ff1156) and a DDR3 SO-DIMM memory module for each FPGA. The two FPGAs are interconnected via high-speed links. A third FPGA, a Spartan-6, is used for board management purposes. Two on-board custom mezzanine connectors provide I/O flexibility for a wide range of front ends. Two mezzanine models exist with $24 \mathrm{ADC}$ channels (12 bit, up to $60 \mathrm{MSa} / \mathrm{s}$ ) and 12 DTCC links on HDMI connectors to interface digital front ends. In-design mezzanines include multi-gigabit optical transceivers with FPGAs. The ATCA-FEC is functionally equivalent to 2 "classic" FEC modules. SRS-ATCA includes rear transition modules (RTM) for multiple GbE, $10 \mathrm{GbE}$ and other I/O connectivity. ATCA backplanes exist with full mesh and star topologies, using $10 \mathrm{~Gb} / \mathrm{s}$ channels.

Besides NEXT-NEW, another application for SRS-ATCA is the micromegas detector readout for the upgrade of the ATLAS muon spectrometer [10].

\section{DAQ and trigger requirements in the NEXT-NEW detector}

NEXT [11] is an underground experiment aimed at searching for neutrinoless double-beta decay that combines an excellent energy resolution with tracking capabilities merging PMTs and SiPMs. NEXT-DEMO, a small-scale demonstrator, was read out using classical SRS. NEXT has adopted SRS-ATCA for its first stage, called NEXT-NEW. This detector (figure 3) is a half scale of the final detector and it will be installed in the Canfranc Underground Laboratory (LSC) using the same platform, gas system and external shielding.

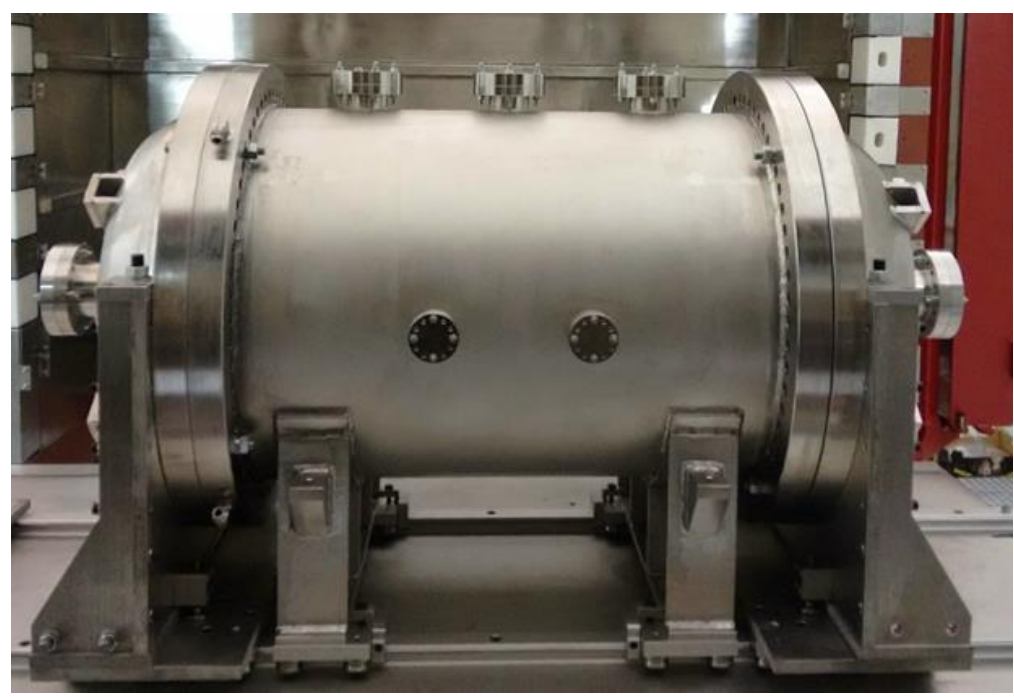

Figure 3. The NEW detector at Canfranc Underground Laboratory (LSC).

The detector is a time-projection chamber filled with $10 \mathrm{~kg}$ of ${ }^{136} \mathrm{Xe}$ gas at 15 bar. Electrons from double-beta decays will drift towards the anode end cap under the effect of an electric field, ionizing other atoms in their movement. Close to the anode, electrons are further accelerated by an additional electric field to produce electroluminescence.

Light received in an array of 1,800 SiPMs in the anode (tracking plane) is used to reconstruct electron tracks in order to reject background events. SiPM sensors are laid out with a 1-cm pitch and grouped in twenty-eight 8x8 arrays. Front-end boards [12] integrate and digitize (12 bit, $1 \mathrm{MSa} / \mathrm{s}$ ) 64 channels, resulting in approx. $1 \mathrm{Mb}$ of data per event per front-end 
board, taking into account the framing overhead. For 28 front-end boards, 1 ms events and a 10 $\mathrm{Hz}$ trigger rate, the SiPM tracking partition generates $35 \mathrm{MByte} / \mathrm{s}$ in raw data mode.

Light produced by electroluminescence is reflected to the energy plane in the cathode end cap where and array of 12 PMTs is used to measure the energy of the event with a resolution better than $1 \%$. PMTs also sense the initial scintillation light produced in the double-beta decay, giving a timestamp and a z-coordinate for the event. PMT signals are digitized at $40 \mathrm{MSa} / \mathrm{s}, 12$ bit. Once digitized and framed, this produces approximately $10 \mathrm{MByte} / \mathrm{s}$ for the $12 \mathrm{PMTs}$ at a $10 \mathrm{~Hz}$ rate.

Dead time must be minimized, suggesting the use of double buffers. These are implemented in the SiPM front-end boards for the tracking plane partition, and in the ATCAFEC's DDR3 memory in the case of the energy plane partition. Events are configurable in length up to $3.2 \mathrm{~ms}$, with a nominal value of $1.6 \mathrm{~ms}$. NEXT-NEW is expected to operate at a 10 $\mathrm{Hz}$ trigger rate produced by background events.

Three different DAQ modes must be supported: (1) raw data mode, (2) Normal Mode 1 ("normal events" are sent zero-suppressed, and "interesting events" are sent in raw mode) and (3) Normal Mode 2 (similar to Normal Mode 1, but the double buffer -and thus the dead time reduction- is only used for "interesting events").

The trigger algorithm [13] must combine information from the first scintillation light as well as from the early total event energy estimation. A trigger is generated if (1) a defined number of PMTs have detected at least a certain number of scintillation photons within a defined time window, and (2) some time after the scintillation, the estimated total integrated energy in a defined time window is within certain upper and lower bounds. All thresholds and levels must be configurable. The trigger algorithm must be implemented on FPGA.

\section{Readout and DAQ Architecture for NEXT-NEW}

Reading out the $28 \mathrm{SiPM}$ front-end boards in the tracking plane partition requires one and a half ATCA-FECs and a total of three digital interface mezzanines (each one having 12 DTCC interfaces over HDMI). Front-end boards work in free-running mode, storing data continuously in a circular buffer. Data are only sent to the ATCA-FEC modules when a timestamped trigger is received.

The energy plane (12 PMTs) is read out with a single 24-channel ADC mezzanine plugged onto half ATCA-FEC module, which sends trigger candidates based on early energy estimations to another half ATCA-FEC (used as trigger module). As a result, three ATCA-FECs are needed to read out the detector's energy and tracking planes and implement the trigger algorithm. Each ATCA-FEC interfaces a DAQ PC via 4 Gigabit Ethernet optical links.

Communication between the trigger module (half ATCA-FEC) and the other DAQ modules should normally make use of high-speed channels in the backplane, be it star or full mesh (both are valid for our application). Implementing the DTCC functionalities in these channels would efficiently address clock, trigger, slow controls and data (trigger candidates) distribution. In an initial stage of operation, in order to ease the porting of the firmware from "classical" SRS to SRS-ATCA, a digital interface mezzanine will be used to create DTCC links over HDMI, which connect to DTCC HDMI sockets in the RTMs in the ATCA-FECs. This mimics the solution used in NEXT-DEMO. A NIM input in the RTM module allows external triggers if needed.

The DAQ PC farm comprises three local data concentrator PCs (each connected to an ATCA-FEC via 4 Gigabit Ethernet optical links), two global data concentrator PCs (for event 
buiding) and a storage PC (equipping a number of hard disks). A Gigabit Ethernet switch interconnects these six PCs. A complete scheme of the NEXT-NEW DAQ and trigger system is shown in figure 4.

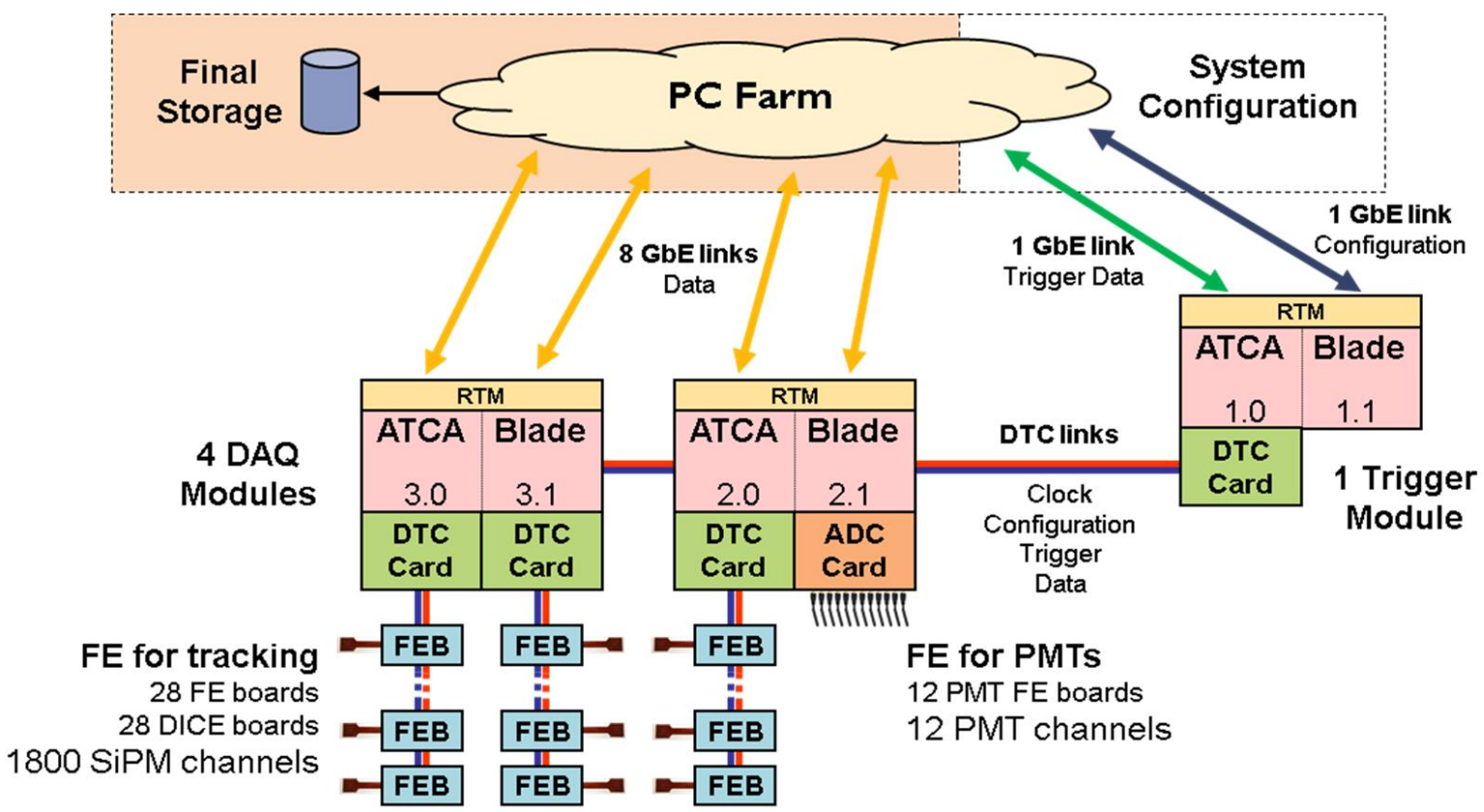

Figure 4. NEXT-NEW DAQ and trigger system architecture.

In a first step, the NEXT-NEW detector, readout and DAQ system, only for the energy plane, was installed and tested at the Canfranc Underground Laboratory during September 2015 (see figure 5). The complete system described in figure 4 has been already successfully validated in laboratory. Installation and initial operation with the NEXT-NEW detector in the Canfranc Underground Laboratory (LSC) is scheduled for November 2015.

\section{Conclusions}

SRS, in its "classic" flavour, is a successful DAQ and trigger solution for our first prototype NEXT-DEMO as well as for many other applications in the CERN RD51 community. Longterm operation of NEXT-NEW and the final NEXT detector in an underground laboratory poses more stringent demands on reliability and performance. Porting SRS to the ATCA standard, using the same key technologies (same FPGAs, functional blocks and protocols), provides an adequate upgrade for the hardware with the additional benefit of an easy migration path for the firmware.

The complete readout, DAQ and trigger system has been validated in laboratory prior to installation in the underground laboratory in November 2015. As early users, we had access to a beta version of the ATCA-FEC module and helped to identify minor design bugs in the course of our tests. NEXT-NEW is, to our knowledge, the first experiment operating entirely on SRSATCA. 


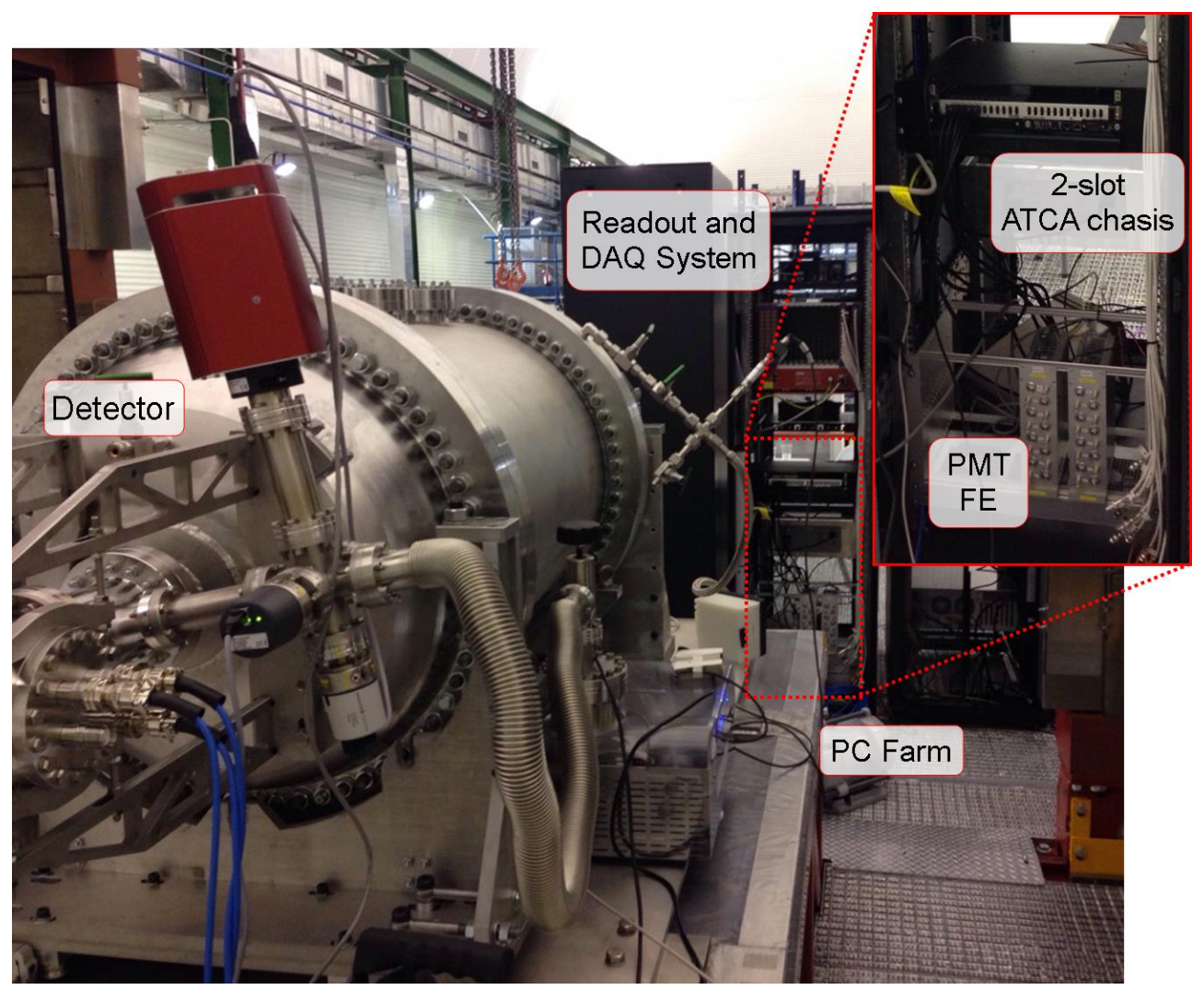

Figure 5. NEXT-NEW detector, readout and DAQ system installation in the Canfranc Underground Laboratory.

\section{Acknowledgments}

The authors would like to acknowledge the collaboration of the membership of the NEXT experiment. The European Commision under the European Research Council 2013 Advanced Grant 339787 - NEXT, the Ministerio de Economía y Competitividad of Spain and FEDER under grants CONSOLIDER-Ingenio 2010 CSD2008-0037 (CUP), FIS2012-37947-C04-04 and FIS2014-53371-C4-4. The Director, Office of Science, Office of Basic Energy Sciences, of the US Department of Energy under contract no. DE-AC02-05CH11231; and the Portuguese FCT and FEDER through the program COMPETE, project PTDC/FIS/103860/2008.

\section{References}

[1] A. D. Oltean Karlsson and B. Martin, ATCA: Its Performance and Application for Real Time Systems, IEEE Trans. Nucl. Sci, vol. 53, no. 3, pp. 688-693, June 2006.

[2] Markus Joos, Interest in xTCA rising at CERN as experiments plan for DAQ upgrade, http://xtcasystems.com/articles/interest-xtca-rising-cern-experiments-plan-daq-upgrade, March 2013.

[3] B. Spruck et al., The Belle II Pixel Detector Data Acquisition and Reduction System, IEEE Trans. Nucl. Sci., vol. 60, no. 5, pp. 3709-3713, October 2013. 
[4] J. Olsen, T. Liu and Y. Okumura, A full mesh ATCA-based general purpose data processing board, 2014 JINST 9 C01041.

[5] S. Martoiu, H. Muller, A. Tarazona and J. Toledo, Development of the scalable readout system for micro-pattern gas detector and other applications, 2013 JINST 8 C03015.

[6] S. Martoiu et al., Front-end electronics for the Scalable Readout System of RD51, IEEE Nucl. Sci. Symp. Med. Imag. Conf. (2011) pp. 2036-2038.

[7] J.Toledo et al., The front-end concentrator card for the RD51 scalable readout system, 2011 JINST $6 \mathrm{C} 11028$.

[8] A. Tarazona et al., A point-to-point link for data, trigger, clock and control over copper or fibre, 2014 JINST 9 T06004.

[9] V. Álvarez et al., Initial results of NEXT-DEMO, a large scale prototype of the NEXT-100 experiment, 2013 JINST 8 P04002.

[10] A. Zibell, Micromegas detectors for the upgrade of the ATLAS muon spectrometer, 2014 JINST 9 C08013.

[11] V. Álvarez et al., NEXT-100 Technical Design Report (TDR), 2012 JINST 7 T06001.

[12] J. Rodríguez et al., The front-end electronics for the 1.8-kchannel SiPM tracking plane in the NEW detector, 2015 JINST 10 C01025.

[13] R. Esteve et al, The trigger system in the NEXT-DEMO detector, 2012 JINST 7 C12001. 